\title{
Looking beyond COVID-19 as a pandemic
}

This article was published on $12 \mathrm{Apr}$ 2021 at www.hkmj.org.

\author{
YL Lau *, MD (Hon) \\ Department of Paediatrics and Adolescent Medicine, The University of Hong Kong, Queen Mary Hospital, Hong Kong \\ *Corresponding author: lauylung@hku.hk
}

Hong Kong Med J 2021;27:88-9

https://doi.org/10.12809/hkmj215114

By early April 2021, coronavirus disease 2019 (COVID-19) pandemic had resulted in over 132 million cases with an overall case fatality rate of $2.2 \%$ globally. ${ }^{1}$ The infection fatality rate is lower, perhaps as low as $0.2 \% .^{2}$ However, although the infection fatality rate is near zero for those aged $<40$ years, it increases rapidly for people aged $>60$ years to reach over $25 \%$ at the extreme end of old age. ${ }^{3}$ A population-based seroprevalence study in Wuhan, China, suggested that $>80 \%$ of antibodypositive individuals were asymptomatic. ${ }^{4}$ In Hong Kong, $>25 \%$ of reported COVID-19 cases have been asymptomatic; among children and young adults the proportion is even higher at $>40 \%$ (personal communication, Centre for Health Protection).

Considering the low infection fatality rates, some countries opted initially for acquiring herd immunity through natural infection; however that has led to high numbers of cases and deaths, potentially resulting in healthcare system collapse. ${ }^{5}$ At present, universal whole-population vaccination is considered the only viable option to move the world out of the current pandemic, with non-pharmaceutical interventions being deployed as a stop-gap measure to contain the scale of the pandemic until effective herd immunity is achieved through vaccination. ${ }^{6}$ The socio-economic cost of some non-pharmaceutical interventions is extremely high, resulting in damage to the economy, increasing unemployment rates, and other collateral morbidities, such as depression and delayed diagnosis of severe diseases leading to poorer prognosis. Many other indirect impacts on health, especially in children and older adults, are still to be delineated. Therefore, the speed to achieve herd immunity regionally and globally will become a critical factor to minimise the direct and indirect harms caused by the COVID-19 pandemic.

Because of this, enormous collaborative efforts have gone into developing COVID-19 vaccines at unprecedented speeds. ${ }^{7}$ The usual regulatory pathways have been compressed to allow emergency use of such vaccines despite the availability of only preliminary data from the first 3 to 4 months of phase 3 studies. This has been justified using the argument that the benefits of using these novel vaccines far outweigh the potential harms.

There has been unprecedented international collaboration among scientists, doctors, and pharmaceutical companies to produce candidate vaccines, including mRNA, adenoviral vector, and inactivated whole virus vaccines, and to trial those candidates, with some successfully approved by different national regulatory authorities for emergency use. In contrast, subsequent steps to implement universal vaccination has exposed frailties in human societies even in a pandemic, such as rivalry among vaccine producers, distribution logistics of vaccines among nations, and vaccine inequity between the rich and poor nations. These issues have been compounded by mistrust between citizens and governments, fuelling vaccine hesitancy. In reality, the pandemic will not end if only a handful of rich and capable nations achieve herd immunity. So what are the future scenarios for this divided world and humanity? Let us look at ourselves first.

In Hong Kong, with a densely packed population that is highly connected, both physically and electronically, adequate vaccine supply for the whole population has been secured. Therefore, we should be in an enviable position among the first few regions to reach herd immunity. Instead, we remain far from this goal because of vaccine hesitancy, internal conflict, and mistrust. There is no easy solution except to insist on basic principles such as voluntary vaccination, freedom to choose a preferred vaccine, transparency, and effective communication of all matters related to vaccines and the vaccination programme, including adverse events following immunisation, in order to dispel misinformation. Moreover, our own healthcare professionals who hold the primary responsibility to care for the health of our citizens should be empowered to educate the public regarding the reality and purpose of the vaccination rollout. These healthcare professionals should have the confidence to explain and counsel citizens to exercise a rational choice. Everyone should understand the post hoc ergo propter hoc fallacy; in this context, an adverse event following vaccination is not necessarily caused by the vaccination. Nevertheless, a vigorous pharmacovigilance risk assessment system has to be in place to detect signal of adverse events that could be causally linked with these novel vaccines, such as the rare complication of embolic and thrombotic events in young population with the AstraZeneca ChAdOx1-S recombinant COVID-19 vaccine. ${ }^{8}$

To be tolerant of choosing which vaccine to 
use will lead to balance and healing of our divided society. Diversity of choice among different types of vaccines is preferred to having only one type available, because there are many unknowns, including the long-term performance of each vaccine. The longterm vaccination strategy is far from being fixed, as new knowledge is being accrued daily. Maintaining diversity will allow room to adapt to this uncertainty. Lastly, it is now becoming clear that COVID-19 will likely stay with us as an endemic disease and cannot be eradicated globally or eliminated regionally at national levels, because of suboptimal vaccine uptake, emergence of spike variants that escape immunity, and the limited duration of sterilising humoral immunity after either natural infection or vaccination..$^{9-12}$ In contrast, memory $\mathrm{T}$ cell immunity could be long-lasting and reduce disease severity when reinfection occurs. ${ }^{12}$ Therefore the goal of the vaccination programme is not to interrupt all infections, which would be unrealistic, but rather to prevent severe COVID-19. With the attainment of herd immunity through vaccination, primary COVID-19 will then likely occur during the first 5 years of life, with mild symptoms; partially transmissible reinfection may occur throughout life to boost immune memory, as in the other four common human coronaviruses. ${ }^{3}$ This will render COVID-19 as a common cold rather than a severe disease. This is the preferred and most likely future scenario, rather than global eradication, which is unrealistic and would demand repeated annual vaccinations.

\section{Author contributions}

The author contributed to the editorial, approved the final version for publication, and takes responsibility for its accuracy and integrity.

\section{Disclosures}

YL Lau is a member of the Advisory Panel on COVID-19 Vaccines; and the chairman of the Scientific Committee on Vaccine Preventable Diseases for the Hong Kong SAR Government.

\section{References}

1. Our World in Data. Available from: https://ourworldindata. org. Accessed 8 Apr 2021.

2. Randolph HE, Barreiro LB. Herd immunity: understanding COVID-19. Immunity 2020;52:737-41.

3. Lavine JS, Bjornstad ON, Antia R. Immunological characteristics govern the transition of COVID-19 to endemicity. Science 2021;371:741-5.

4. He Z, Ren L, Yang J, et al. Seroprevalence and humoral immune durability of anti-SARS-CoV-2 antibodies in Wuhan, China: a longitudinal, population-level, crosssectional study. Lancet 2021;397:1075-84.

5. Sridhar D, Gurdasani D. Herd immunity by infection is not an option. Science 2021;371:230-1.

6. Baker MG, Wilson N, Blakely T. Elimination could be the optimal response strategy for covid-19 and other emerging pandemic diseases. BMJ 2020;371:m4907.

7. Jeyanathan M, Afkhami S, Smaill F, Miller MS, Lichty BD, Xing Z. Immunological considerations for COVID-19 vaccine strategies. Nat Rev Immunol 2020;20:61532.

8. European Medicines Agency Pharmacovigilance Risk Assessment Committee. Signal assessment report on embolic and thrombotic events (SMQ) with COVID19 Vaccine (ChAdOx1-S [recombinant])-COVID-19 Vaccine AstraZeneca (other viral vaccines). EPITT no: 19683. 24 March 2021. Available from: https://www.ema. europa.eu/en/documents/prac-recommendation/signalassessment-report-embolic-thrombotic-events-smqcovid-19-vaccine-chadox1-s-recombinant-covid_en.pdf. Accessed 8 Apr 2021.

9. Seow J, Graham C, Merrick B, et al. Longitudinal observation and decline of neutralizing antibody responses in the three months following SARS-CoV-2 infection in humans. Nat Microbiol 2020;5:1598-607.

10. Chia WN, Zhu F, Ong SW, et al. Dynamics of SARS-CoV-2 neutralising antibody responses and duration of immunity: a longitudinal study. Lancet Microbe 2021 Mar 23. Epub ahead of print

11. Dan JM, Mateus J, Kato Y, et al. Immunological memory to SARS-CoV-2 assessed for up to 8 months after infection. Science 2021;371:eabf4063.

12. Bonifacius A, Tischer-Zimmermann S, Dragon AC, et al. COVID-19 immune signatures reveal stable antiviral $\mathrm{T}$ cell function despite declining humoral responses. Immunity 2021;54:340-54.e6. 